\title{
Trichostrongylus probolurus
}

National Cancer Institute

\section{Source}

National Cancer Institute. Trichostrongylus probolurus. NCI Thesaurus. Code C125971.

A species of parasitic roundworms in the family Trichostrongylidae that most often infests sheep, goats, and camels. 